\title{
ANATOMICAL BASIS OF SPECIFIC CONNECTIONS BETWEEN SENSORY AXONS AND MOTOR NEURONS IN THE BRACHIAL SPINAL CORD OF THE BULLFROG ${ }^{1}$
}

\author{
JEFF W. LICHTMAN, ${ }^{2}$ SONAL JHAVERI, ${ }^{3}$ AND ERIC FRANK ${ }^{4}$ \\ Department of Neurobiology, Harvard Medical School, Boston, Massachusetts 02115 \\ Received October 10, 1983; Revised January 26, 1984; Accepted January 30, 1984
}

\begin{abstract}
The anatomical basis for the specificity of the monosynaptic stretch reflex has been studied in the brachial spinal cord of bullfrogs. Sensory axons from the triceps brachii muscle innervate the corresponding triceps motoneurons but do not innervate two types of unrelated motoneurons (subscapularis and pectoralis) (Lichtman, J. W., and E. Frank (1984) J. Neurosci. 4: 1745-1753). Retrograde labeling of these three types of motoneurons with horseradish peroxidase (HRP) demonstrated that their cell bodies had overlapping distributions in the lateral motor column, and their dendrites all occupied the same region of the dorsal horn. In addition, triceps sensory axons aborized extensively in the dorsal horn throughout the brachial spinal cord, with no obvious predilection for the region of the triceps motoneurons. Thus, the physiological specificity of these sensory-motor connections was not apparent from the anatomical location of the sensory or motor neurons. However, by injecting single pairs of related or unrelated sensory and motor cells with HRP, we found that related pairs formed anatomical contacts with each other more frequently than unrelated sensory-motor pairs did. These observations suggest that the specificity of these connections is most likely the result of local interactions between sensory and motor processes.
\end{abstract}

In the bullfrog, as in other vertebrates, the connections between stretch-sensitive sensory axons and motoneurons are highly specific (Cruce, 1974; Tamarova, 1977; Frank and Westerfield, 1982). In the preceding paper (Lichtman and Frank, 1984), we have shown that individual sensory axons within a particular head of the triceps muscle provide monosynaptic input to almost all of the triceps motoneurons projecting to the same head (homonymous connections) and that these sensory axons innervate approximately half of the synergistic motoneurons that project to the other triceps heads (heteronymous connections). These high projection frequencies

\footnotetext{
${ }^{1}$ It is a pleasure to acknowledge useful suggestions and criticism from P. C. Jackson and D. Sah, and expert technical assistance from J. Gagliardi, M. Nastuk, Y. Pearlson, M. Peloquin, and E. Wu. This work was supported by National Institutes of Health Granls NS 14451 and NS 20859 and National Science Foundation Grant BNS-80-21605 to E. F.

${ }^{2}$ To whom correspondence should be sent, at his present address: Department of Physiology and Biophysics, Washington University School of Medicine, 660 South Euclid Avenue, St. Louis, MO 63110.

${ }^{3}$ Present address: Department of Psychology and Brain Science, Massachusetts Institute of Technology, Cambridge, MA 02139.

${ }^{4}$ Present address: Department of Neurobiology and Physiology, Northwestern University, Evanston, IL 60201.
}

are in marked contrast to the rare occurences of monosynaptic connections between triceps sensory axons and at least two other types of motoneurons in the vicinity of the triceps motoneuron pool.

How is it that a sensory axon can establish synapses with virtually all of the motoneurons in one pool and innervate virtually none in some other overlapping pool? One possibility is that the dendrites of the various types of motoneurons are spatially segregated in the cord. Thus, a rigid topographic mapping of sensory fibers only onto certain locations in the spinal cord could result in a proper matching between afferent axons and appropriate target cells. Alternatively, dendrites of various types of motoneurons may be intermingled rather than spatially distinct. The proper pairing of each sensory fiber and motoneuron could then result from local interactions during development leading to selective synaptogenesis between appropriate pre- and postsynaptic partners.

The aim of this study was to determine the anatomical basis of the physiological specificity of these sensorymotor connections. We found that, although sensory axon terminals are in close proximity to the dendritic arbors of appropriate and inappropriate motoneurons, they make many more anatomical contacts with appropriate motoneurons. These results suggest that local 
interactions between pre- and postsynaptic elements are necessary to explain the observed specificity.

\section{Materials and Methods}

Adult and juvenile bullfrogs (Rana catesbeiana) obtained from Amphibians of North America (Nashville, TN) or metamorphosed in our laboratory were employed in this study. The nose-to-tail length of the animals was 4 to $7 \mathrm{~cm}$.

Horseradish peroxidase (HRP) backfills of sensory axons and motoneurons. Sensory and motoneurons were labeled retrogradely by application of HRP to peripheral nerves (Kristensson and Olsson, 1971). Frogs were anesthetized by immersion in $0.2 \%$ tricaine methanesulfonate (Sigma Chemical Co., St. Louis, MO). Cuffs containing a concentrated solution of HRP (Boehringer Manheim, type I) with $2 \%$ lysolecithin (Sigma) were applied to the triceps, subscapularis, or pectoralis nerves according to the method described by Frank et al. (1980). Following survival periods of 1 to 3 weeks, animals were perfused transcardially with $1.25 \%$ glutaraldehyde and $1.0 \%$ paraformaldehyde in $0.1 \mathrm{M}$ phosphate buffer ( $\mathrm{pH} 7.4)$. The brachial spinal cord was sectioned at $50 \mu \mathrm{m}$ in the transverse or parasagittal plane, and the sections were processed according to the method of Mesulam (1978), utilizing tetramethyl benzidine as a substrate for the enzyme reaction.

Intracellular HRP injection of motoneurons and sensory axons. Isolated preparations of the brachial spinal cord from adult bullfrogs were used for these studies (see Frank and Westerfield, 1982; Lichtman and Frank, 1984 for details). The connections of peripheral nerves to the cord were maintained intact on one side. The following peripheral nerves were routinely dissected with the cord: ulnaris, radialis, triceps (medial, internal, and external branches), subscapularis, pectoralis, and deltoideus.

Microelectrodes with tips etched for $30 \mathrm{sec}$ in boiling $0.5 \mathrm{M}$ potassium citrate were backfilled with a solution of $25 \%$ HRP (Boehringer Manheim type I) in $0.4 \mathrm{M}$ potassium acetate buffered with $0.2 \mathrm{M}$ Tris to $\mathrm{pH}$ 7.4. Motoneurons were impaled and identified by antidromic activation (Frank and Westerfield, 1982; Lichtman and Frank, 1984). HRP was injected for 10 to 15 min with 1 to $2 \mathrm{nA}$ of depolarizing current in 50 -msec pulses at 5 to $10 \mathrm{~Hz}$. Sensory axons were impaled and injected in the dorsal root entry zone. The afferents were identified by intracellular recording of action potentials while stimulating the nerve to a particular arm muscle or by stretching the muscle. The injection method was the same as for motoneurons except that the injection time was prolonged to 30 to $60 \mathrm{~min}$.

Following injection of sensory axons and motoneurons, the isolated spinal cord was maintained in frog Ringer's solution at $14^{\circ} \mathrm{C}$ for 8 to $10 \mathrm{hr}$ and then fixed in a solution of $1.25 \%$ glutaraldehyde, $1 \%$ paraformaldehyde buffered to $\mathrm{pH} 7.4$ with $0.1 \mathrm{M}$ phosphate buffer. Sections were cut on a cryomicrotome at a thickness of $80 \mu \mathrm{m}$ in the transverse plane, and the HRP was visualized using diaminobenzidine with cobalt intensification (Adams, 1977). Injected sensory axons and motoneurons were reconstructed with the aid of camera lucida drawings. In two cases, a computer-aided reconstruction of a pair of sensory and motor neurons was made (Gilbert and Wiesel, 1983). The three-dimensionally reconstructed pairs were then used to make the stereo pairs shown in Figures 9 and 10.

\section{Results}

The results describe the spatial arrangement of three motoneuron pools (triceps, subscapularis, and pectoralis) and the anatomical relationship of these pools to the three corresponding groups of sensory axons which innervate the same muscles. We have studied the anatomical relationships within the triceps system in great detail since there is already quantitative physiological data available (Frank and Westerfield, 1982; Lichtman and Frank, 1984). Subscapularis and pectoralis motoneuron pools were well suited for comparison with the triceps system since triceps sensory axons discriminate among these motoneuronal types, yet all three types of motoneurons are located in the same region of the spinal cord.

\section{Spatial distribution of motoneurons and sensory axons}

Pattern of motoneuronal organization. Each sensory axon in the triceps nerve exhibits a high probability of forming monosynaptic connections with every triceps motoneuron, but a low likelihood of projecting monosynaptically onto a non-triceps motoneuron (Lichtman and Frank, 1984). One way that such a selective projection might be brought about is by spatial segregation of the different pools of motoneurons. To investigate this possibility, we first determined the anatomical position of motoneuronal somata by labeling motoneurons with HRP applied to individual muscle nerves.

In the rostrocaudal axis the motoneurons of the subscapularis and pectoralis pools overlap with those in the triceps pool (Fig. 1). Similarly, in the mediolateral and dorsoventral axes, many of the motoneurons from the three pools are intermingled (Fig. 2). Although pectoralis motoneurons tend to be more ventral and medial than the other two pools, there is no clear cytoarchitectonic delineation between the three motoneuronal groups, and they overlap considerably. The location of motoneuronal cell bodies thus provided no clues as to the basis of the impressive physiological specificity.

Pattern of dendritic arborization. To assess the spatial organization of dendrites of motoneurons, we made intracellular injections of HRP into several individual but closely spaced motoneurons projecting to the same muscle (Fig. 3). We were able to reconstruct the complete motoneuronal dendritic trees which spread over rostrocaudal distances of 600 to $800 \mu \mathrm{m}$. Most of the dendrites were found in the dorsolateral, lateral, and ventrolateral directions from the cell somata. In fact, the lateral dendrites traverse through the white matter and reach the pial surface where they turn and course subpially, parallel to the surface of the cord, for 50 to $100 \mu \mathrm{m}$. Dorsomedially, where the dendrites enter the intermediate region of the gray matter (target area of muscle afferent axons, often called "ventral neuropil" in the frog) (Jhaveri and Frank, 1983), the density of dendrites is somewhat reduced. Ventromedially, in the region of the ventral fun- 
SPINAL CORD

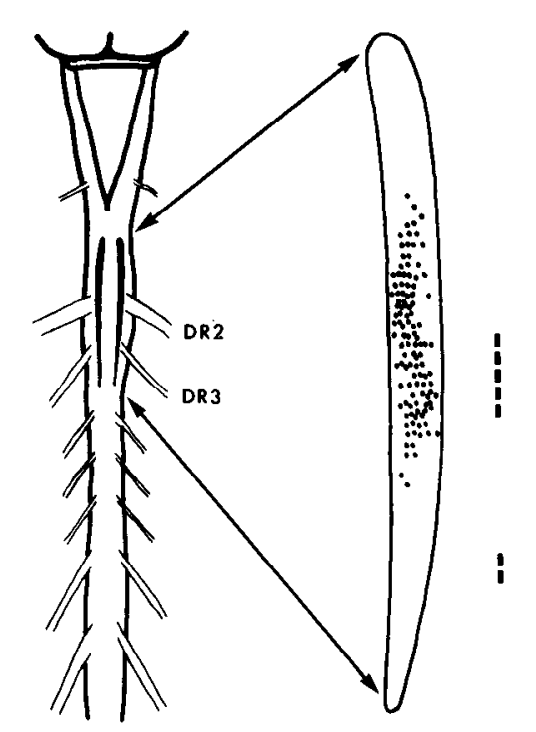

Subscapularis
BRACHIAL MOTOR

COLUMN

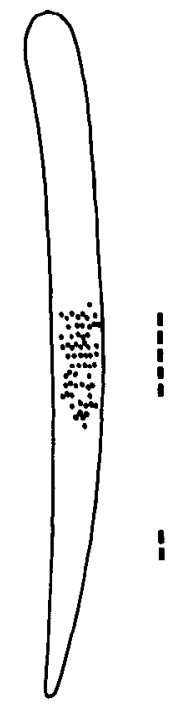

Triceps

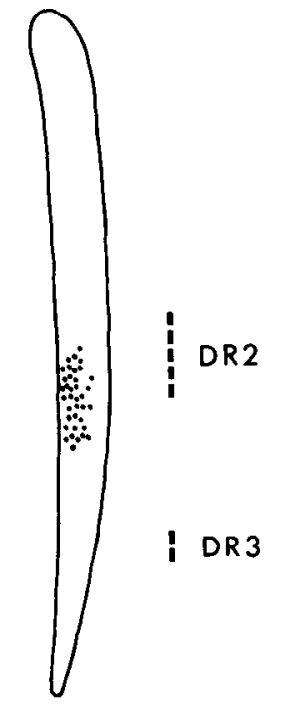

Pectoralis

\section{MOTONEURON POOLS}

Figure 1. Schematic drawing of a bullfrog's central nervous system, dorsal view. The lateral motor column in the brachial region is shaded in the left-hand drawing and is redrawn at higher magnification to the right. All of the sensory and motor axons of the subscapularis, triceps, and pectoralis nerves course in the second spinal nerve, whose dorsal root is indicated (DR2). The enlarged drawings (right) illustrate the location of subscapularis, triceps, and pectoralis motor pools. Individual motoneuronal somata are indicated by black dots and were determined by labeling each of the three muscle nerves with HRP. The triceps motor pool overlaps extensively with the pools of the other two types of motoneurons.

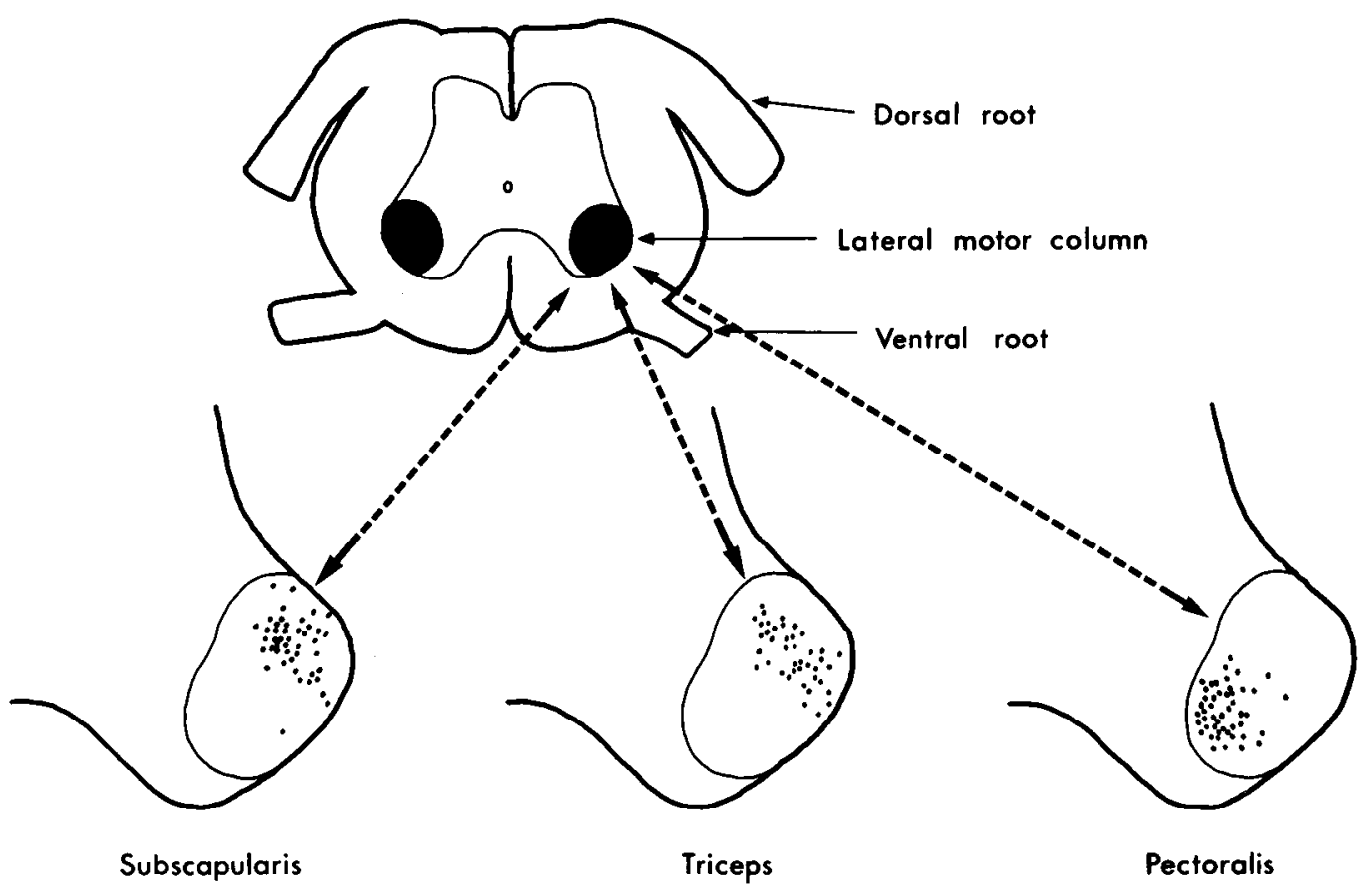

Figure 2. Schematic drawing of the spinal cord in transverse section at the level of the second dorsal root. The ventral horn is enlarged in the lower drawings to show the location of subscapularis, triceps, and pectoralis motoneurons which were labeled by applying HRP to each of the muscle nerves. All labeled motoneurons from each of the pools that are within the rostrocaudal extent of the triceps motor pool are shown; thus the figure includes all triceps and pectoralis motoneurons but only about half of the subscapularis motoneurons (refer to Fig. 1). 

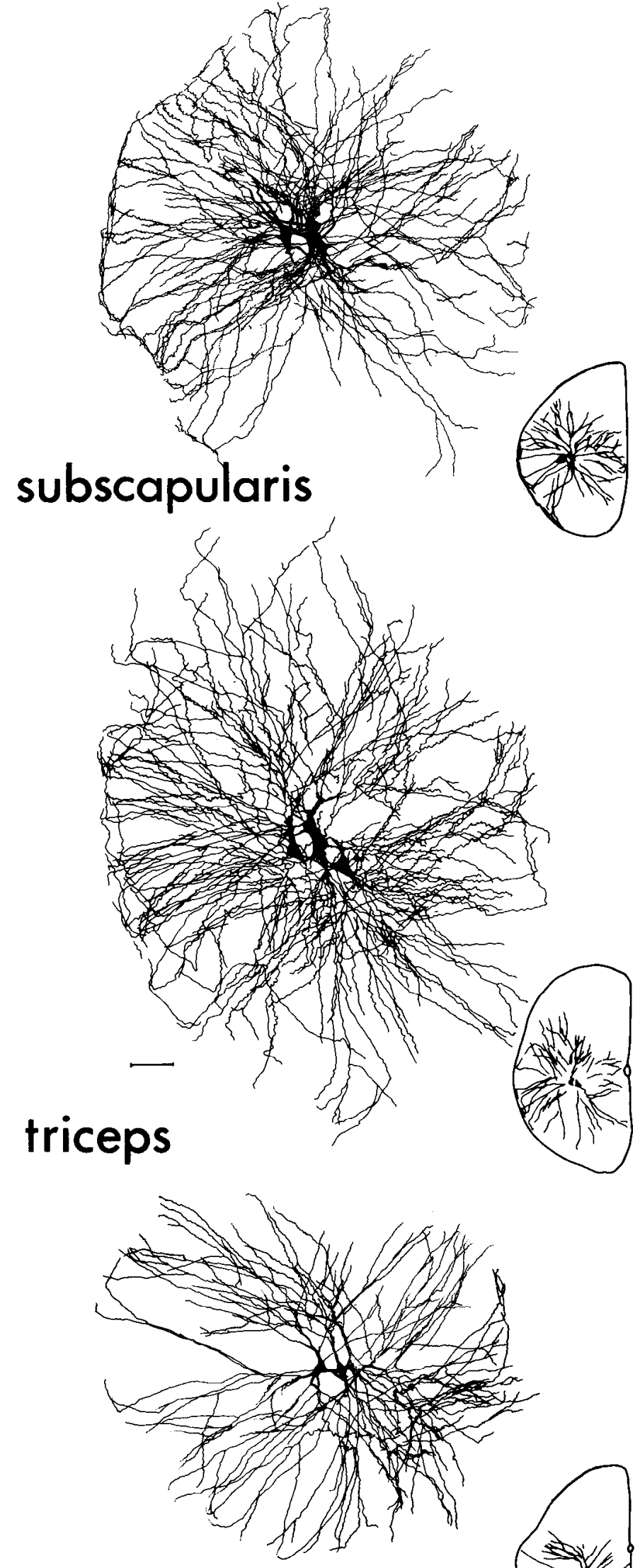

pectoralis

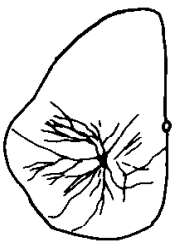

Figure 3. Camera lucida reconstructions, from $80-\mu \mathrm{m}$ serial sections, of clusters of physiologically identified, HRP-injected iculus, the dendritic projection field is the most sparse. The important observation for the purpose of our study is that this pattern of dendritic spread holds true for motoneuronal dendrites from the triceps, subscapularis, and pectoralis motoneuronal pools. Thus, there was no evidence of dendritic segregation between the different sets of motoneurons.

The spatial arrangement of motoneuronal cell bodies and their dendrites therefore provides no anatomical basis for the specificity of connections between sensory axons and motoneurons. The triceps, subscapularis, and pectoralis pools are widely overlapping.

Pattern of sensory afferent projection. To examine whether sensory axons innervating the triceps, subscapularis, and pectoralis muscles are spatially segregated from each other in the spinal cord gray matter, we examined spinal cords from frogs in which pairs of these three muscle nerves had been separately backfilled with HRP on opposite sides of the animal. Labeled sensory axons from the three muscles are generally alike. Each set enters the spinal cord through the same dorsal root (the second) and bifurcates to course rostrally and caudally in the dorsal columns, over the entire extent of the brachial spinal cord (Fig. 4). Some fibers even project beyond the motor column and extend rostrally past the obex (Jhaveri and Frank, 1983).

Ventrally oriented collaterals of these muscle sensory fibers branch off from the axons in the dorsal columns and descend into the spinal cord gray matter at various rostrocaudal sites to ramify in the ventral neuropil region. In sagittal and transverse sections, these axons are seen to descend to the ventral neuropil singly or in small groups. There is no general tendency for neighboring ventral collaterals from the same nerve to fasciculate with each other (see also Scheibel and Scheibel, 1969). The maximal density of terminal arborization is located in a region about $500 \mu \mathrm{m}$ on either side of the dorsal root entry zone. Within this region, sensory axons from muscle nerves ramify over the entire mediolateral extent of the intermediate zone (Ebbesson, 1976; Fig. 5). In sections rostral and caudal to this critical zone, the afferent terminal field becomes considerably narrower.

There was no obvious difference in the mediolateral extent of sensory arbors among the three types of muscle sensory axons we examined (Fig. 5). This effectively rules out an explanation of sensory-motor specificity based on a systematic difference in the location of different classes of sensory terminals.

To investigate this pattern of projection at the singlecell level further, we injected individual triceps sensory axons and reconstructed their pattern of projection (Fig. 6). Each sensory axon we studied $(n=20)$ coursed widely in the brachial spinal cord, going well rostral and caudal of the three motoneuron pools. In fact, ventral collaterals branched off the parent axon in the dorsal column without an obvious predilection for any specific region. For

motoneurons from individual pools. At the bottom right of each group of cells is an orientation drawing showing a hemi-cord with the motoneurons and major dendrites. Scale bar $=100$ $\mu \mathrm{m}$. 


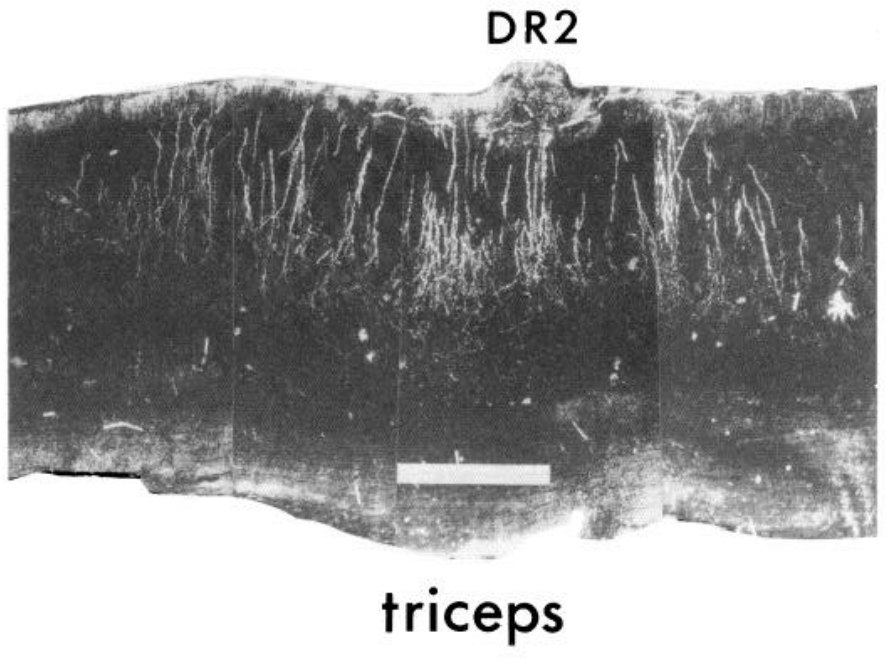

DR 2

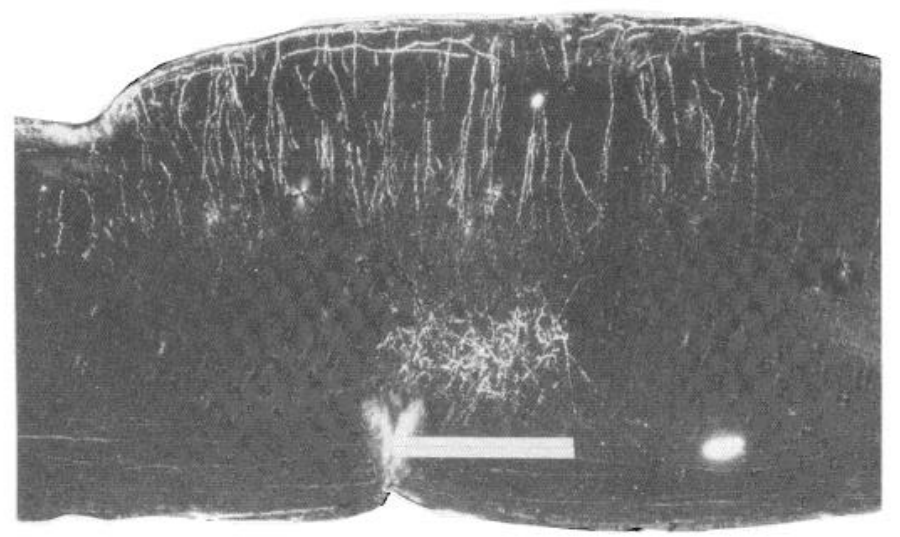

pectoralis

Figure 4. Photomicrographs of the sensory axons of two muscles viewed in parasaggital sections of the brachial spinal cord. Triceps (top) and pectoralis (bottom) muscle nerves were labeled with HRP in separate animals. Dorsal is to the top and anterior to the right of each photograph. Ventrally oriented collaterals of labeled sensory axons are visible descending to the region of the ventral neuropil. The white horizontal bar indicates the position and extent of the triceps (top) and pectoralis (bottom) motoneuron pools. The sensory axons from both muscles ramify throughout the rostrocaudal extent of the brachial spinal cord well beyond the boundaries of the corresponding motor pool. DR2, second dorsal root. Scale bar $=500$ $\mu \mathrm{m}$. Tetramethyl benzidine (TMB) reaction was photographed with cross-polarized illumination.

example, the medial triceps sensory axon shown in Figure 6 had 18 ventral collaterals of which only 3 were in a position to innervate triceps motoneuronal dendrites. The ventral collaterals emerged from the dorsal column axon at an average interval of $300 \mu \mathrm{m}$, but there did not seem to be any systematic periodicity. Although triceps sensory axons certainly do innervate interneurons and a few motoneurons outside the region of the triceps motoneuron pool, it was surprising to us that the density of collaterals (i.e., the number of collaterals per millimeter of spinal cord) was no higher inside this pool than elsewhere.

However, we did find differences in the extent of terminal arborizations of the ventral collaterals from triceps sensory axons within the triceps pool compared to outside the pool (Fig. 6). The collaterals within the pool were more highly branched with greater numbers of boutons. The greater complexity of these terminal ramifications may reflect the fact that these collaterals are innervating a larger number of motoneurons (see Lichtman and Frank, 1984). As mentioned previously, however, dendrites of many other motoneurons (including subscapularis and pectoralis) are also located within this region, but the triceps axons selectively avoid innervating non-triceps dendrites. The anatomical pattern of arborization of single afferent axons thus does not provide any obvious clues to this physiological specificity.

\section{Location of sensory-motor connections}

Since neither the deployment of motoneuronal dendrites nor the pattern of afferent projection separately provided evidence suggesting how sensory axons selectively innervate only certain motoneurons, we examined the relationship between pairs of individual sensory axons and motoneurons. Two types of sensory-motor pairs were studied: 8 homonymous pairs (e.g., a medial triceps sensory axon and a medial triceps motoneuron) in which the likelihood of monosynaptic contact was about $95 \%$ (Lichtman and Frank, 1984) and 10 unrelated pairs (a medial triceps sensory axon and a pectoralis or subscapularis motoneuron located within the region of the triceps pool) in which there was a $>95 \%$ probability that the axon would not innervate the motoneuron.

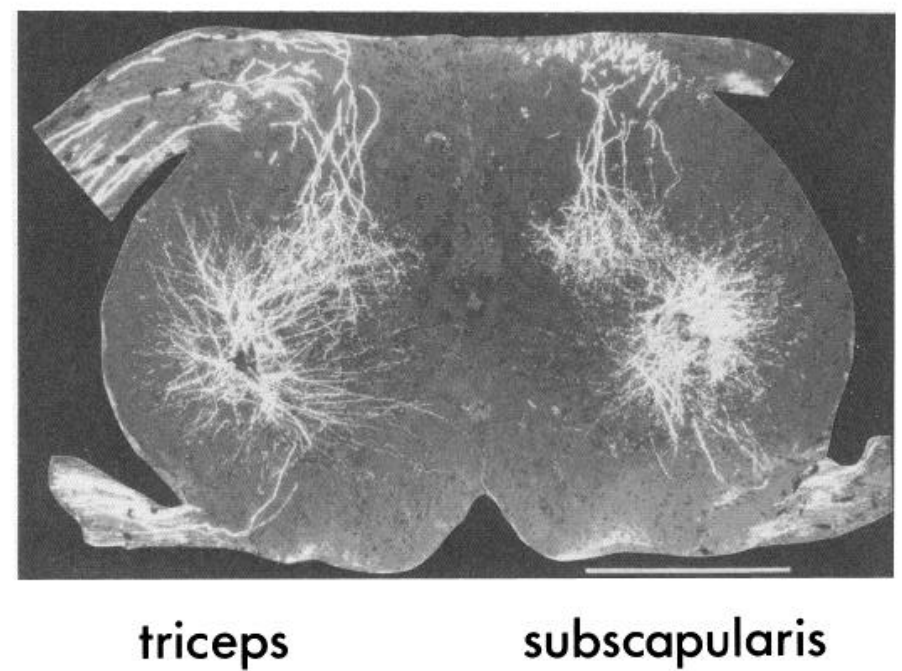

Figure 5. Photomicrograph of muscle sensory axons and motoneurons viewed in a transverse section of brachial spinal cord. The left triceps and right subscapularis muscle nerves were labeled with HRP in the same animal. The arborization of sensory axons from both muscles occurs within the same cross-sectional area of the spinal cord. There is no systematic anatomical difference in the two arborizations although physiologically their connectivity is very dissimilar. Dorsal is up. Scale bar $=500 \mu \mathrm{m}$. Polarization optics of TMB reaction. 

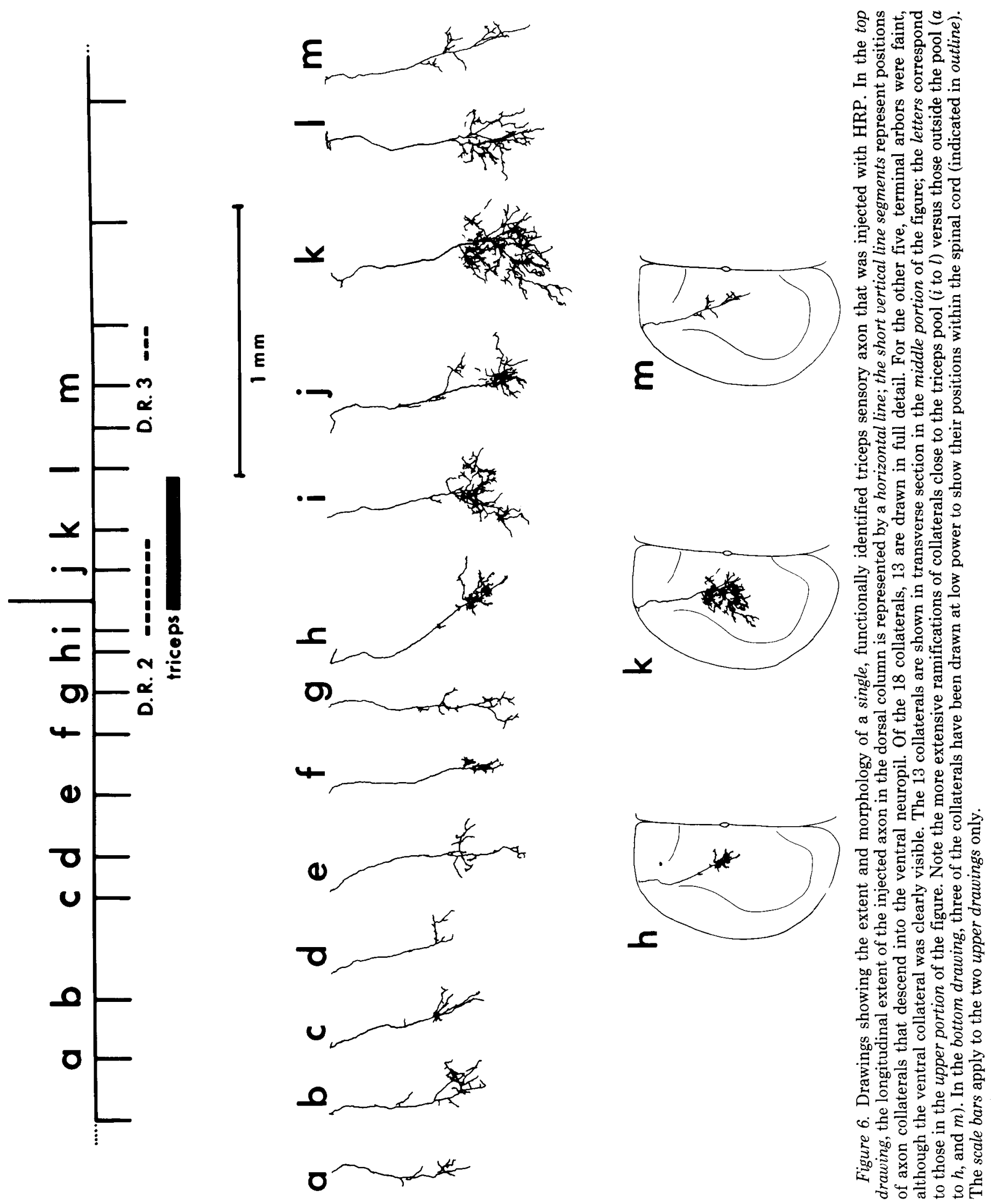
Homonymous pairs. All of the homonymous pairs shared features in common. First, each sensory axon had one collateral that descended into close proximity to the dorsomedially oriented dendrite (dendrite 1 of Bregman and Cruce, 1980) of the homonymous motoneuron (Fig. 9 ). Second, at a number of places (mean, 10.2; range, 2 to $21 ; n=8$ ), the varicosities on the sensory axons were in close apposition to the homonymous motoneuron dendrite (Fig. 7). These are the putative synaptic connections between sensory axons and motoneurons. The places of close apposition were not all located on the same dendritic branch. For two of the eight pairs, the sensory axon reached and appeared to make contact with the cell body (see also Grantyn et al., 1982).

Unrelated pairs. At low magnification the unrelated sensory-motor pairs looked similar to the homonymous pairs in the relationship of the sensory axons to the dorsomedial motoneuron dendrites (Fig. 8). The dendrites appeared to enter into and intermingle with the terminal ramifications of the axons. In contrast to the homonymous pairs, however, the axon was usually not immediately adjacent to the dendrites. As a result, the number of close appositions (putative synaptic contacts) between a sensory axon and unrelated motoneuron dendrite was less than for the homonymous pairs (mean, 1.2 ; range 0 to $2 ; n=10$ ).
In order to illustrate this difference graphically, we reconstructed, in three dimensions, one homonymous and one unrelated sensory-motor pair with the help of a computer graphics system. The computer then produced physiological estimate: the number ascertained anatomstereo pairs for each sensory-motor set. The homonymous sensory-motor pair is shown in Figure 9 and the unrelated pair in Figure 10. The difference between the homonymous and the unrelated pairs becomes obvious when viewed in three dimensions: dendrites of triceps motoneurons are insinuated into and totally interwoven with the terminal arbor of a triceps (homonymous) sensory axon. In contrast, although dendrites of a subscapularis motoneuron approach and share the same general target region as a triceps (unrelated) sensory collateral, the two are not intermingled. Each appears to maintain its own spatial "microdomain." This observation from the three-dimensional stereo pairs serves to explain the fewer numbers of contacts shared by an unrelated pair when compared to the homonymous pair (see above).

\section{Discussion}

The work presented here describes the anatomical relation of triceps muscle sensory axons with two different sorts of motoneurons: homonymous cells with which they make frequent connections and nearby motoneu-
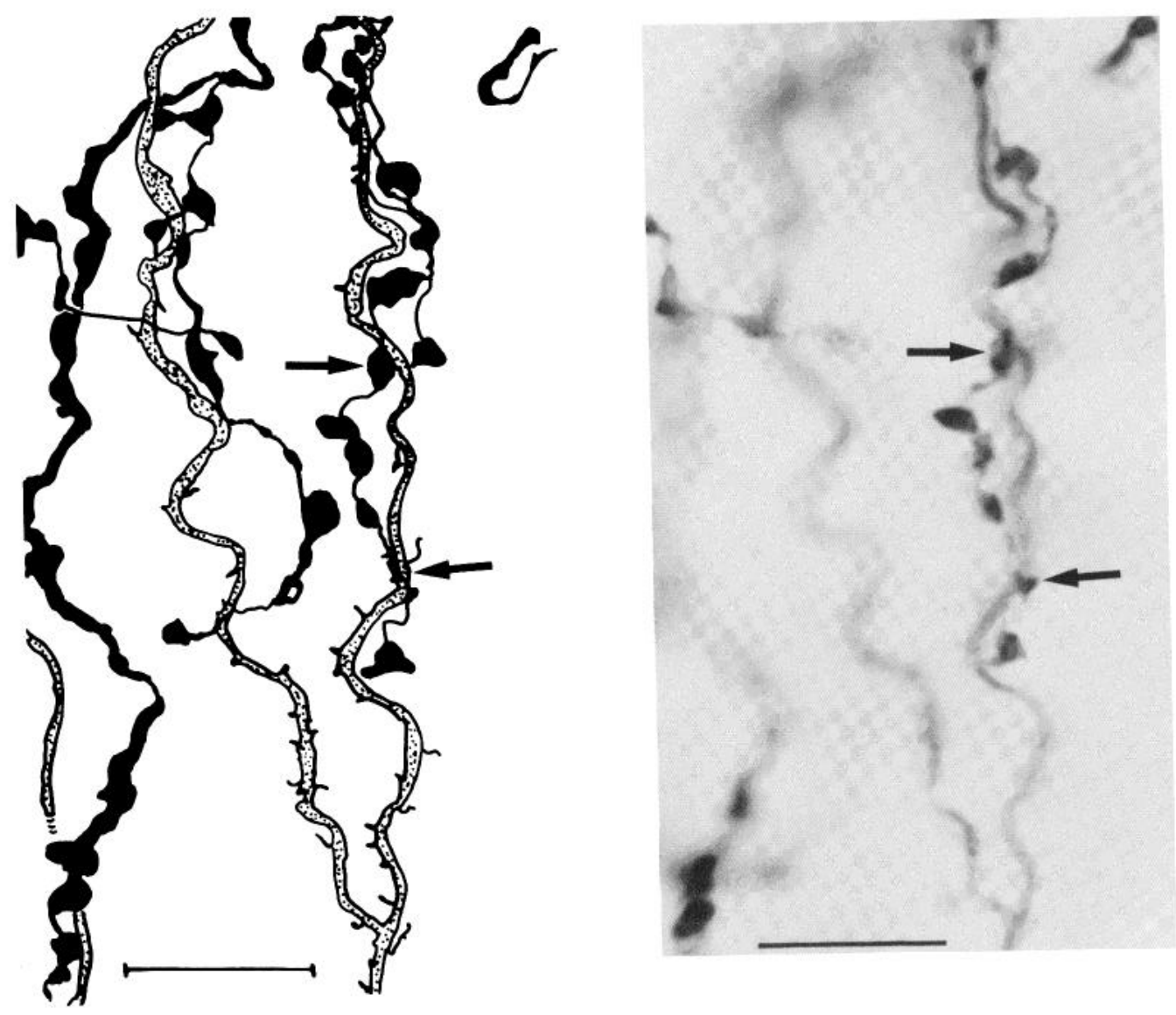

Figure 7. Photomicrograph and corresponding camera lucida drawing of a portion of triceps sensory axon (with varicosities) in close apposition to a dorsomedial dendrite (wavy processes with spines) of a homonymous triceps motoneuron. Two of the varicosities (arrows) appear to be in direct contact with the dendrite. Scale bar $=10 \mu \mathrm{m}$. 


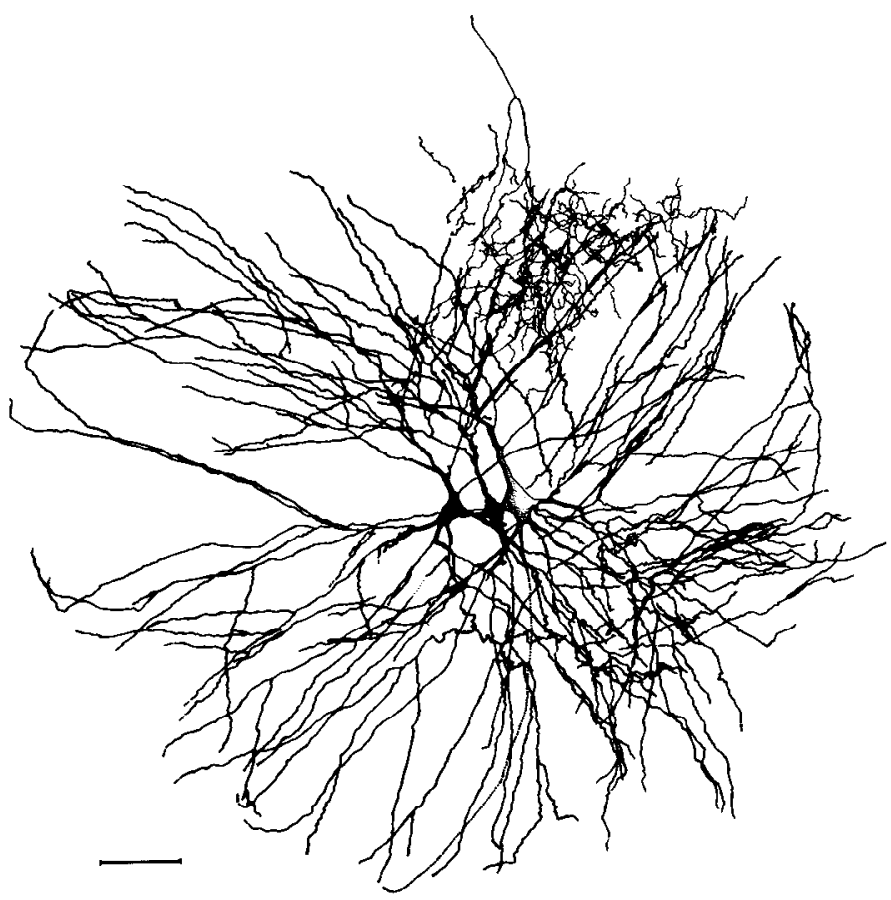

Figure 8. Camera lucida drawing of unrelated sensory-motor pairs. A medial triceps sensory axon and three pectoralis motoneurons were injected with HRP. When the drawing is viewed at this power and in two dimensions, the sensory arbor appears to ovelap extensively with the motoneuronal dendrites even though the sensory fiber almost certainly did not innervate these motoneurons. Scale bar $=100 \mu \mathrm{m}$.

rons which they innervate only infrequently (Lichtman and Frank, 1984). The results indicate that specificity in the sensory-motor connections of the bullfrog's spinal cord is most apparent when looking at the level of resolution of individual motoneurons and sensory axons. Thus sensory axons seem to come into close apposition with appropriate motoneuronal dendrites but not with inappropriate dendrites, even when all of the dendrites and sensory axons occupy the same region. These results are discussed in relation to the physiological findings in the previous paper (Lichtman and Frank, 1984) and in relation to comparable studies of this synaptic pathway in frogs and cats by other laboratories.

Location of synaptic contacts. Synaptic contacts between muscle sensory fibers and motoneurons occur primarily in the intermediate region of the gray matter; this is the major area of termination of the muscle sensory afferents (Jhaveri and Frank, 1983). In only two of all of our pairs of injected cells did the sensory fiber project ventrally as far as the region of motoneuronal somata. Shapovalov and co-workers (Grantyn et al., 1982; Motorina et al., 1982) report a similar terminal distribution in the lumbar spinal cord of the frog, although several of their injected fibers penetrated more deeply into the ventral horn. In one of their cell pairs, Motorina et al. (1982) found that half of the contacts apparently were made on the soma, but in most of their cases the proportion of somatic contacts was lower. In the cat, there is general agreement that most of the sensory terminals are also on dendrites; only about $10 \%$ are somatic (Brown and Fyffe, 1978, 1981; Burke et al., 1979; Ishizuka et al., 1979). As reported by Bregman and Cruce (1980), motoneurons in the frog's spinal cord have only one primary dendrite projecting into the intermediate zone, so that all sensory contacts are made on branches of this one dendrite, whereas in the cat an individual sensory fiber can contact several different primary dendrites (Rurke et al., 1979; Ishizuka et al., 1979; Brown and Fyffe, 1981). Each axon typically establishes connections on a number of higher-order dendritic branches, an observation common to all studies of this system made to date, both in frogs and in cats (Burke et al., 1979, 1980; Ishizuka et al., 1979; Brown and Fyffe, 1981; Grantyn et al., 1982). The anatomical basis of this reflex pathway in frogs is thus remarkably similar to the analogous pathway in cats.

Number and efficacy of synaptic contacts. From physiological studies of single afferent input to motoneurons an estimate was made of the average number of synapses between a sensory axon and a homonymous motoneuron in the frog (Lichtman and Frank, 1984). These estimates assumed that the smallest synaptic potentials observed between a sensory axon and motoneuron were due to the average synaptic potential elicited by one bouton. Thus, if the smallest potentials are $40 \mu \mathrm{V}$ and the average homonymous connections about $200 \mu \mathrm{V}$, then an average of 5 boutons of contact should exist between a sensorymotor pair. The number of potential contacts between individual homonymous sensory and motor neurons was found to range from 2 to 21 (average of 10.2). Although somewhat high, this range is in good agreement with the physiological estimate: the number ascertained anatomically is almost certainly an overestimate since every site of close apposition between a bouton and a dendrite observed with the light microscope need not be a synaptic contact.

By dividing the amplitude of the average single-fiber synaptic potential $(200 \mu \mathrm{V})$ by the observed number of contacts (10.2), we calculate that each anatomical contact produces an average EPSP of about $20 \mu \mathrm{V}$. This number can be compared with that derived from analogous experiments in both the frog and the cat. Shapovalov and co-workers (Grantyn et al., 1982; Motorina et al., 1982) recently measured the average synaptic potentials associated with four sensory-motor pairs injected with HRP in the lumbar spinal cord of the frog. In those four cases, the ratios were $20,18,10$, and $3.2 \mu \mathrm{V} /$ contact. Similarly, in the cat, if one combines the physiological results of Scott and Mendell (1976) for homonymous EPSPs in soleus and medial gastrocnemius motoneurons (143 and $95 \mu \mathrm{V}$ ) with measurements by Glenn et al. (1982) of the numbers of homonymous contacts for these cell types, the ratios are $18 \mu \mathrm{V} /$ contact and $22 \mu \mathrm{V} /$ contact, respectively. The (probably fortuitously) close quantitative correspondence between these amphibian and mammalian systems suggests that a common mechanism may be regulating the physiological strength of individual synaptic contacts.

The low number of triceps sensory boutons in anatomical contact with unrelated motoneurons also correlates well with our physiological results. The physiological projection was virtually zero (Lichtman and Frank, 

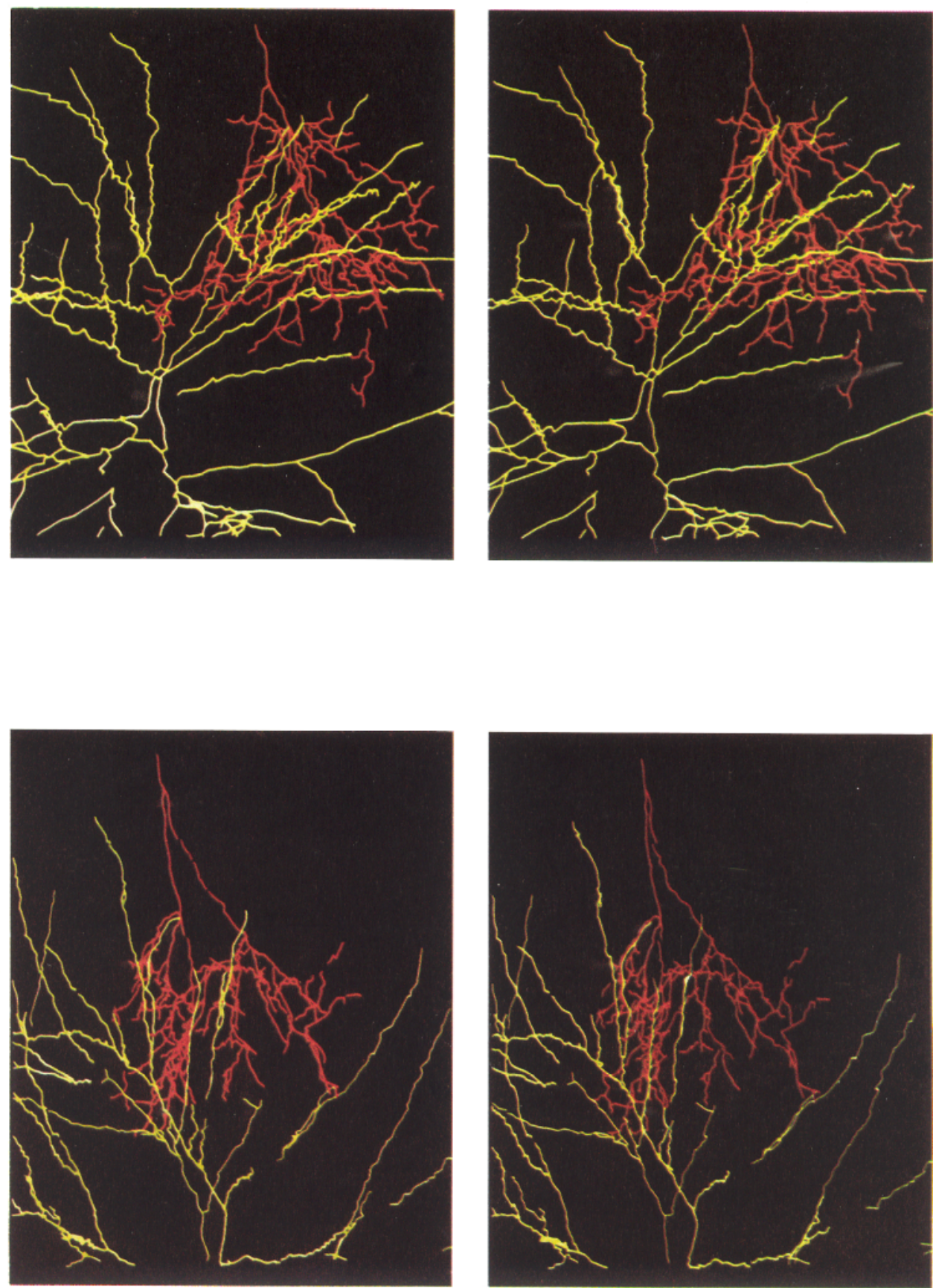

Figure 9. Reconstruction of a medial triceps sensory axon and homonymous medial triceps motoneuron that were injected with HRP. A stereo pair of the region of sensory-motor overlap, made by computer reconstruction, is shown in the transverse plane. When observed in three dimensions (right eye views right figure; left eye views left figure), the sensory axons (red) can be seen intermingling with and contacting the motoneuronal dendrites (yellow). Dorsal is up, medial to the right. The black squares are $\sim 500 \mu \mathrm{m}$ wide.

Figure 10. Relationship of a triceps sensory axon and an unrelated, subscapularis motoneuron, both injected with HRP. The sensory and motor cells appear to overlap when viewed in two dimensions. In the three-dimensional stereo pair shown here, however, one can see that portions of the axonal arbor (red) lie just rostral (behind) or caudal (in front) of the motoneuronal dendrites (yellow), and there are virtually no points at which the cells come into close contact with each other (right eye views right figure; left eye views left figure). The pair is shown in transverse section, dorsal up, medial to the right. The black squares are $\sim 500 \mu \mathrm{m}$ wide.

1984); anatomically we saw between 0 and 2 (average of 1.2) triceps boutons touching dendrites of subscapularis or pectoralis motoneurons. This result suggests that the strength of a synaptic interaction between a muscle sensory and motor neuron may be regulated by the number of anatomical synaptic contacts. There is no need in this system to postulate that the physiological effectiveness of each synaptic terminal is different for appropriate versus inappropriate connections.

Basis of sensory-motor specificity. The striking topographic maps found in some parts of the vertebrate central nervous system raise the possibility that topographic mapping of axons onto postsynaptic targets can account for a major portion of neural specificity. In the 
spinal cord of the frog, however, topography cannot explain the specificity of sensory-motor connections described here. Sensory axons ramify throughout the brachial spinal cord without an obvious predilection to send collaterals into the region where their homonymous motor pool is located. Yet the collaterals that are juxtaposed with the dendrites of appropriate motoneurons have a richer terminal arbor. Moreover, even within this region of rich arborization, synaptic contacts are formed only on appropriate, not inappropriate, motoneurons. These observations are most consistent with a view of neural specificity in which there is a cellular recognition between appropriate synaptic partners rather than a view in which specificity is the result of guidance of sensory axons to a particular place where their preferred targets are waiting. The specificity is thus similar to that found in autonomic ganglia (Lichtman et al., 1979) in which cells of any one class are randomly distributed among other ganglion cells.

In the cat, topography may be more important in determining the specificity of sensory-motor synapses. The longitudinal extent of the motor column is much greater than in the frog, and different species of motoneurons are often located in anatomically distinct regions. Lüscher et al. (1980) have demonstrated that single Ia afferents make stronger projections to motoneurons in their spinal segment of entry than elsewhere, and Zengel et al. (1983) have reported a similar finding for group II afferents. However, Mendell and co-workers (Scott and Mendell, 1976; Nelson and Mendell, 1978) showed that, even in the cat, topography is not sufficient to explain the specificity of these connections; a "species" specificity must also be involved that operates to distinguish among different species of motoneurons (see also Burke et al., 1980; Brown and Fyffe, 1981).

Our results suggest that "species" specificity, by itself, can account for a very high level of synaptic specificity, although they certainly do not argue against the importance of topography as another important determinant of neuronal connectivity. The basis for the cellular affinities between pre- and postsynaptic cells that underlie this "species" specificity remains an intriguing question.

\section{References}

Adams, J. C. (1977) Technical considerations on the use of horseradish peroxidaes as a neuronal marker. Neuroscience 2: 141-145.

Bregman, B. S., and W. L. R. Cruce (1980) Normal dendritic morphology of frog spinal motoneurons: A Golgi study. J. Comp. Neurol. 193: 1035-1045.

Brown, A. G., and R. E. W. Fyffe (1978) The morphology of group Ia afferent fibre collaterals in the spinal cord of the cat. J. Physiol. (Lond.) 274: 111-127.

Brown, A. G., and R. E. W. Fyffe (1981) Direct observations on the contacts made between Ia afferent fibres and $\alpha$ motoneurones in the cat's lumbrosacral spinal cord. J. Physiol. (Lond.) 313: 121-140.

Burke, R. E., B. Walmsley, and J. A. Hodgson (1979) HRP anatomy of group Ia afferent contacts on alpha motoneurones. Brain Res. 160: 347-352.

Burke, R. E., M. J. Pinter, A. Lev-Tov, and M. J. O‘Donovan (1980) Anatomy of monosynaptic contacts from group Ia afferents to defined types of extensor $\alpha$-motoneurons in the cat. Soc. Neurosci. Abstr. 6: 713.

Cruce, W. L. R. (1974) Supraspinal input to hindlimb motoneurons in lumbar spinal cord of the frog, Rana catesbeiana. J. Neurophvsiol. 37: 691704.
Ebbesson, S. O. E. (1976) Morphology of the spinal cord. In Frog Neurobiology, R. Llinás and W. Precht, eds., pp. 679706, Springer-Verlag, Berlin.

Frank, E., and M. Westerfield (1982) Synaptic organization of sensory and motor neurones innervating triceps brachii muscles in the bullfrog. J. Physiol. (Lond.) 324: 479-494.

Frank, E., W. A. Harris, and M. B. Kennedy (1980) Lysophosphatidyl choline facilitates labelling of CNS projections with horseradish peroxidase. J. Neurosic. Methods 2: 183-189.

Gilbert, C. D., and T. N. Wiesel (1983) Clustered intrinsic connections in the cat visual cu.tex. J. Neurosci. 3: 11161133.

Glenn, L. L., R. E. Burke, J. W. Fleshman, and A. Lev-Tov (1982) Estimates of electrotonic distance of group Ia contacts on cat $\alpha$-motoneurons: An HRP morphological study. Soc. Neurosci. Abstr. 8: 995.

Grantyn, R., A. I. Shapovalov, and B. I. Shiriaev (1982) Combined morphological and electrophysiological description of connections between single primary afferent fibres and individual motoneurons in the frog spinal cord. Exp. Brain Res. 48: 459-462.

Ishizuka, N., H. Mannen, T. Hongo, and S. Sasaki (1979) Trajectory of group Ia afferent fibers stained with horseradish peroxidase in the lumbrosacral spinal cord of the cat: Three-dimensional reconstructions from serial sections. J. Comp. Neurol. 186: 189-212.

Jhaveri, S., and E. Frank (1983) Central projections of the brachial nerve in bullfrogs: Muscle and cutaneous afferents project to different regions of the spinal cord. J. Comp. Neurol. 221: 304-312.

Kristensson, K., and Y. Olsson (1971) Retrograde axonal transport of protein. Brain Res. 29: 363-365.

Lichtman, J. W., and E. Frank (1984) Physiological evidence for specificity of synaptic connections between individual sensory and motor neurons in the brachial spinal cord of the bullfrog. J. Neurosci. 4: 1745-1753.

Lichtman, J. W., D. Purves, and J. W. Yip (1979) On the purpose of selective innervation of guinea-pig superior cervical ganglion cells. J. Physiol. (Lond.) 292: 69-84.

Lüscher, H. R., P. Ruenzel, and E. Henneman (1980) Topographic distribution of terminals of Ia and group II fibers in spinal cord, as revealed by postsynaptic population potentials. J. Neurophysiol. 43: 968-985.

Mesulam, M. (1978) Tetramethyl benzidine for horseradish peroxidase neurohistochemistry: A non-carcinogenic blue reaction product with superior sensitivity for visualizing neural afferents and efferents. J. Histochem. Cytochem. 26: 106117.

Motorina, M. V., Z. A. Tamarova, I. A. Shapovalov, and B. I. Shiriaev (1982) Investigation of synaptic connections between frog spinal cord primary afferents and motoneurons by means of HRP intracellular injection. Neurophysiologia 14: $60-68$.

Nelson, S. G., and L. M. Mendell (1978) Projection of single knee flexor la fibers to homonymous and heteronymous motoneurons. J. Neurophysiol. 41: 778-787.

Scheibel, M. E., and A. B. Scheibel (1969) Terminal axonal patterns in cat spinal cord. III. Primary afferent collaterals. Brain Res. 13: 417-443.

Scott, J. G., and L. M. Mendell (1976) Individual EPSP's produced by single triceps surae la afferent fibers in homonymous and heteronymous motoneurons. J. Neurophysiol. 39: 679-692.

Tamarova, Z. A. (1977) Excitatory postsynaptic potentials induced in the frog lumbar motoneurones by muscle and cutaneous nerve stimulation. Sechenov J. Physiol. U. S. S. R. 63: $806-813$.

Zengel, J. E., G. W. Sypert, and J. B. Munson (1983) Relation of single fiber EPSP amplitude in spinal motoneurons to location of afferent entry. Soc. Neurosci. Abstr. 9: 862. 